\title{
The Synaptomic Theory of Behavior and Brain Disease
}

\author{
Seth G.N. Grant \\ Genes to Cognition Program, Centre for Clinical Brain Sciences, Edinburgh University, \\ Edinburgh BioQuarter, Edinburgh EH16 4SB, United Kingdom \\ Correspondence: seth.grant@ed.ac.uk
}

\begin{abstract}
The purpose of this article is to outline a new molecular and synaptic theory of behavior called the "synaptomic theory," named because it is centered on the synaptome - the complement of synapses in the brain. Synaptomic theory posits that synapses are structures of high molecular complexity and vast diversity that are observable in maps of the brain and that these synaptome maps are fundamental to behavior. Synaptome maps are a means of writing or storing information that can be retrieved by the patterns of activity that stimulate synapses. Synaptome maps have the capacity to store large amounts of information, including multiple representations within the same map. The dynamic properties of synapses allow synaptome maps to store dynamic sequences of representations that could serve to program behavioral sequences. Synaptome maps are genetically programmed and experience-dependent, thereby storing innate and learned behaviors, respectively. Although learning occurs by modification of the synapse proteome, it does not require long-term potentiation (LTP) of synaptic weight or growth of new synapses, and the theory predicts that LTP modulates information recall. The spatial architecture of synaptome maps arise from an underlying molecular hierarchy linking the genome to the supramolecular assembly of proteins into complexes and supercomplexes. This molecular hierarchy can explain how genome evolution results in the behavioral repertoire of the organism. Mutations disrupting this molecular hierarchy change the architecture of synaptome maps, potentially accounting for the behavioral phenotypes associated with neurological and psychiatric disorders.
\end{abstract}

The synaptomic theory is set against the backdrop of the classical connectionist theory of behavior, which has dominated neuroscience for the past century and was born in an era before any knowledge of the molecular biology of the brain (Tanzi 1893; Hebb 1949; Kandel 1976). In contrast, synaptomic theory is based on findings collected during the past 30 years of research into the molecular biology of synapses.

The emphasis of the connectionist theory of behavior is on the anatomical circuitry and the role of stable synaptic strength or synaptic "weight." The main features of the connectionist theory are (i) neurons are the fundamental biological building blocks; (ii) neurons are branched, electrically active cells with axons sending and dendrites receiving the nerve impulses; (iii) the synapse is the junction that connects the axon terminal of one neuron to the dendrite of another neuron; (iv) each behavior uses a circuit made up of stable connected neurons; and (v) learning occurs by an increase in the stable synaptic strength between neurons. The term "connectome" has become popular in recent years to describe the complete anatomical circuitry in the brain, whereas the "synaptome" refers to the complete set of molecular subtypes of synapses in the brain (Zhu et al. 2018).

The synaptomic theory centers on the molecular properties of synapses, their diversity, and their capacity to process information in temporal patterns of neural activity (Zhu et al. 2018). The main features of the synaptomic theory are (i) different types and subtypes of synapses are defined by their proteome composition, and the location of these synapses is described in synaptome maps; (ii) synapse diversity and its organization into synaptome maps is a mechanism of storing information (rather than synaptic weight in the connectionist theory); (iii) the information that is stored in the molecular composition of synapses can be recalled or retrieved by the dynamic response of synapses to patterns of neural activity; (iv) the protein composition and its physical organization into complexes and supercomplexes in synapses are the building blocks of the synaptome and are programmed by genetic mechanisms; and (v) the complexes and supercomplexes are molecular machines that integrate, and therefore read, the temporally encoded information in patterns of neural impulses.

The connectionist theory has its roots in neuroanatomy and electrophysiology, whereas the synaptomic theory arises from studies of the molecular biology of the postsynaptic terminal of excitatory synapses. The reason why the postsynaptic terminal of excitatory synapses must be fundamentally important in behavior is that it is the first point on the neuron's surface where information is received and processed. The information arrives in the form of pulses of neurotransmitter from the adjacent presynaptic terminal and it is the temporal patterns of these pulses that is the currency or "neural code" for information transfer in nervous systems (Adrian 1928). The postsynaptic terminal of mammalian excitatory synapses is characterized by a protrusion from the dendrite, called a dendritic spine, and this houses the proteins collectively known as the postsynaptic proteome (PSP). Here, I will briefly summarize six insights from our studies of the PSP that set the stage for the synaptomic theory.

(C) 2018 Grant. This article is distributed under the terms of the Creative Commons Attribution License, which permits unrestricted reuse and redistribution provided that the original author and source are credited. 


\section{The PSP Has High Molecular Complexity}

The proteome of the postsynaptic terminal of vertebrate excitatory synapses has an inordinately high complexity, with more than 1000 highly conserved proteins encoded by as many genes (Husi et al. 2000; Peng et al. 2004; Collins et al. 2005, 2006; Emes et al. 2008; Bayés et al. 2011, 2012, 2017; Emes and Grant 2011; Distler et al. 2014). The actual protein complexity of the postsynaptic terminal of excitatory synapses is 100 -fold more than was expected based on the molecular requirements of the connectionist theories in the 1990s. Why does the PSP need to be so complex? The many proteins and protein complexes in the PSP perform computations in response to temporal patterns of neurotransmitter pulses and modulate many signaling pathways controlling synaptic strength, structural changes, protein turnover, translation, and transcription (Coba et al. 2009; Kopanitsa et al. 2018). In other words, the synapse should be viewed as a highly complex "computer" and not just the simple connector maintaining synaptic weight as portrayed in the connectionist theory.

\section{The PSP Is Organized into Multiprotein Complexes}

Studies of the physical organization of the PSP show that the many and diverse protein types that it contains are organized within supramolecular multiprotein complexes and supercomplexes (complexes of complexes) (Husi and Grant 2001; Frank et al. 2016, 2017; Frank and Grant 2017). These complexes are complicated molecular machines that are made from the different proteins, and their cognate domains contribute many discrete biochemical properties that produce the overall integrative function of the complexes. The supercomplex family assembled by the scaffold protein PSD95 (referred to as PSD95 supercomplexes or MASC [MAGUK-associated signaling complexes]) are the prototype postsynaptic signaling complex (Husi et al. 2000; Husi and Grant 2001; Fernández et al. 2009, 2017; Frank et al. 2016, 2017; Frank and Grant 2017). These supercomplexes vary in size between one and three megaDaltons and are clustered in the postsynaptic terminal beneath the presynaptic active zone where neurotransmitter is released. PSD95 is a member of the membrane-associated guanylate kinase (MAGUK) family of multidomain scaffold proteins and forms dimers that bind to a diverse range of neurotransmitter receptors, ion channels, signaling enzymes, membrane-spanning adhesion proteins, and cytoskeletal proteins. Using genome engineering in mice, I and the members of my laboratory have systematically modified more than 50 proteins in the PSP, studied their roles in synapse physiology and behavior, and found that PSD95 supercomplex proteins have the strongest phenotypes, attesting to the central functional role of these molecular machines (Komiyama et al. 2018; Kopanitsa et al. 2018).

The hierarchical assembly of individual proteins into complexes and then supercomplexes is not a promiscuous process but is governed by genetic mechanisms (Fig. 1; Frank et al. 2016, 2017; Frank and Grant 2017). Those governing supramolecular assembly act in concert with the genetic regulatory mechanisms that determine the level and age of gene expression to control the composition of synapse proteomes in different cell types and brain regions (Skene et al. 2017; Roy et al. 2018a,b). Importantly, these mechanisms determine the combinations of proteins in the supramolecular assemblies, and these in turn are distributed into different synapses to generate synapse diversity (Zhu et al. 2018). Thus, the genetic mechanisms controlling the assembly of multiprotein complexes are fundamental to the diversity of synapses in the brain.

\section{High Synapse Diversity Is Organized into Synaptome Maps}

From a molecular perspective the synapse is a very large structure, and a single dendritic spine of a CA1 pyramidal neuron could house approximately 100,000 individual proteins and at least 100 PSD95 supercomplexes, and spines in cortical neurons are several-fold larger still. At the 2018 Cold Spring Harbor Laboratory Symposium, I

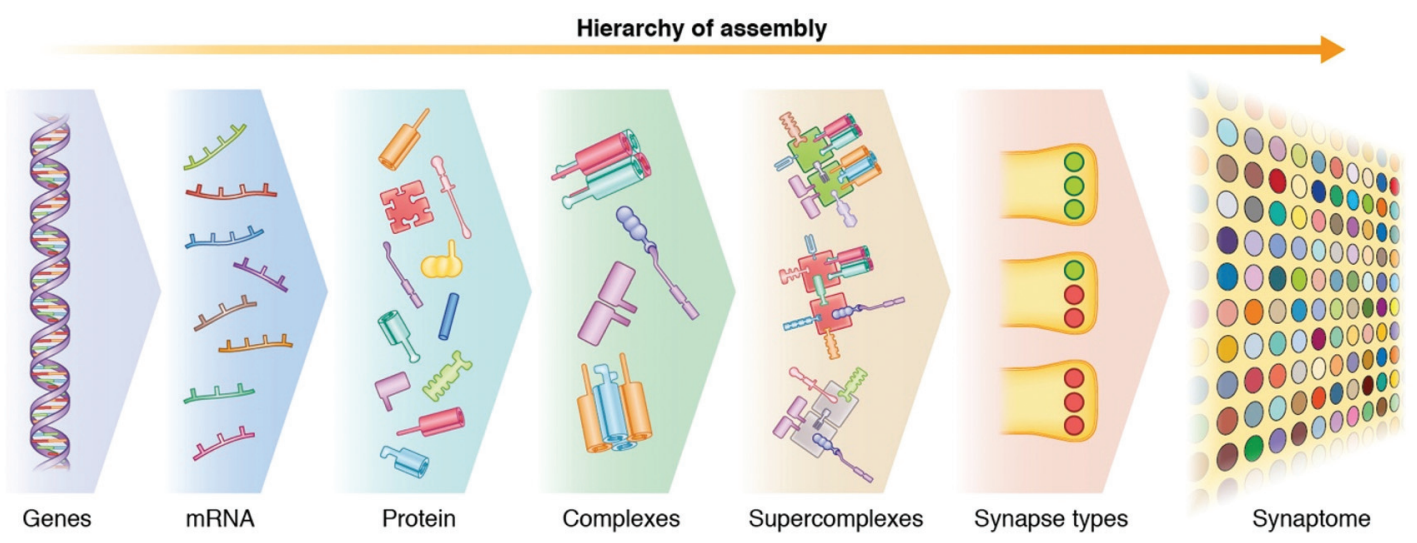

Figure 1. Hierarchical molecular assembly from genome to the synaptome. The genome expresses the transcriptome (RNA), which is translated into the proteome where proteins assemble into complexes (e.g., ion channels) and many of these complexes then assemble into supercomplexes. Both complexes and supercomplexes are distributed into different synapses to produce synapse types, and these are distributed across the brain to produce the synaptome. (Adapted from Zhu et al. 2018.) 


\section{A Dominant subtype maps}

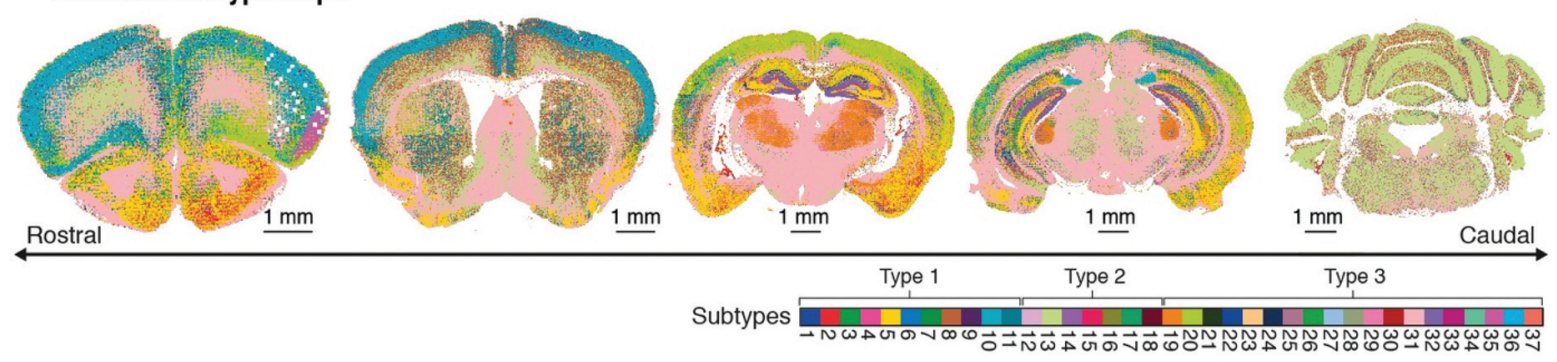

B Synapse diversity maps
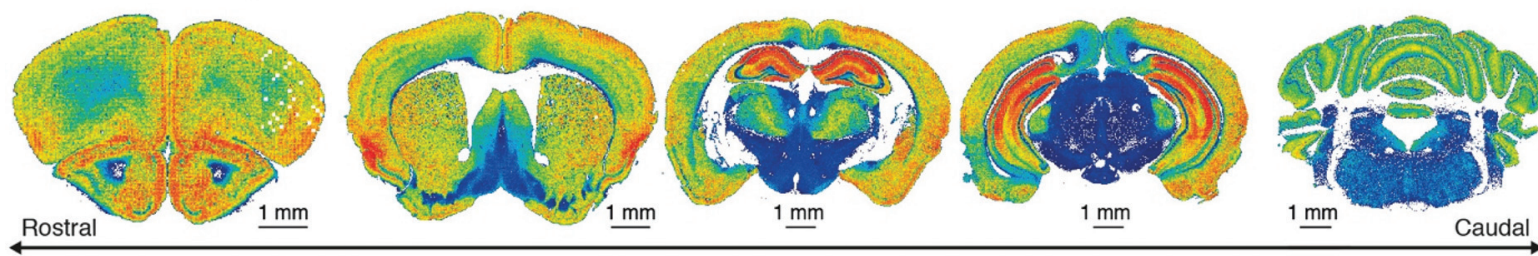

Normalized entropy

$\begin{array}{llllllllllllll}0 & 0.1 & 0.2 & 0.3 & 0.4 & 0.5 & 0.6 & 0.7\end{array}$

Figure 2. Maps of synapse subtypes and diversity of the mouse brain. (A) Synaptome mapping of 37 synapse subtypes in five coronal sections from mouse brain show which subtype is most abundant in each pixel. $(B)$ Maps showing the amount of synapse diversity in each pixel. Note the high diversity in hippocampus and cortex. (Adapted from Zhu et al. 2018.)

presented the first brain-wide single-synapse resolution molecular maps of synapses in the mouse (Zhu et al. 2018). Using fluorescent microscopy of two proteins (PSD95 and SAP102), each labelling a different postsynaptic multiprotein signaling complex, I and the members of my laboratory examined approximately one billion synapses, cataloged them into 37 subtypes, and mapped their distributions across the mouse brain. This was the first description of the whole brain synaptome for any organism. This study revealed an astonishing degree of synapse diversity with the subtypes organized into an architecture at all scales from individual dendrites, cell types, and regions to the global systems-level architecture (Fig. 2). Moreover, the genetic rules that control the hierarchical assembly of proteins into complexes and supercomplexes also controlled the synaptome maps and synapse diversity. Synapse diversity and synaptome maps are exciting new dimensions that have not been considered in the context of the connectionist theory.

\section{Postsynaptic Signaling Complexes Read Time}

Perhaps the most fundamental discovery in neuroscience is that the temporal pattern of nerve impulses encodes all information and is the universal language for neuronal communication (Adrian 1928). The corollary is that there must be mechanisms in the brain that read these patterns of impulses, and the reading mechanism(s) are essential for generating all behavioral responses, perceptions, and thoughts. Synapses are exquisitely sensitive to patterns of nerve impulses and make instantaneous and transient adjustments to the amplitude of the postsynaptic currents triggered by each impulse (Segundo et al. 1963; Markram et al. 1998; Dittman et al. 2000; Abbott and Regehr 2004). This capacity is often described as short-term plasticity
(STP), and proteins in both the pre- and postsynaptic terminal of synapses are required for STP. Particular patterns of nerve impulses can also lead to long-term stable modification of synaptic strength, and the most widely studied form of this plasticity is called long-term potentiation (LTP) (Abbott and Regehr 2004). PSD95 supercomplexes have been shown to control both STP and LTP (Migaud et al. 1998; Komiyama et al. 2002; Kopanitsa et al. 2018), reflecting their importance in reading the information in patterns of nerve impulses and adjusting synaptic strength on timescales from the instantaneous to an hour or more.

The naturally occurring trains of neural impulses are highly complex and can be described using a simplified syntax in which the time interval between a pair of impulses is a fundamental element from which more complex trains, such as bursts of several seconds duration, are built. In a recent study, we showed that different postsynaptic proteins are required for each temporal component of the basic syntax (Kopanitsa et al. 2018). In other words, different PSP proteins are required to read particular patterns of activity (Fig. 3A). This indicates that PSD95 supercomplexes and the overall PSP can extend temporal integration of long trains of activity beyond that of glutamate receptors alone because of the many protein components and their intrinsic and collective dynamics. A further striking result from the large-scale genetic study was that particular combinations of PSP proteins modulated (amplified or attenuated) each of the elements in the basic syntax and that the combinations of proteins in PSD95 supercomplexes were involved in reading all temporal features (Kopanitsa et al. 2018). These findings suggest that the genetic mechanisms that control the expression and supramolecular assembly of combinations of postsynaptic proteins ultimately control the capacity of synapses to detect and discriminate patterns of neural activity. 
A

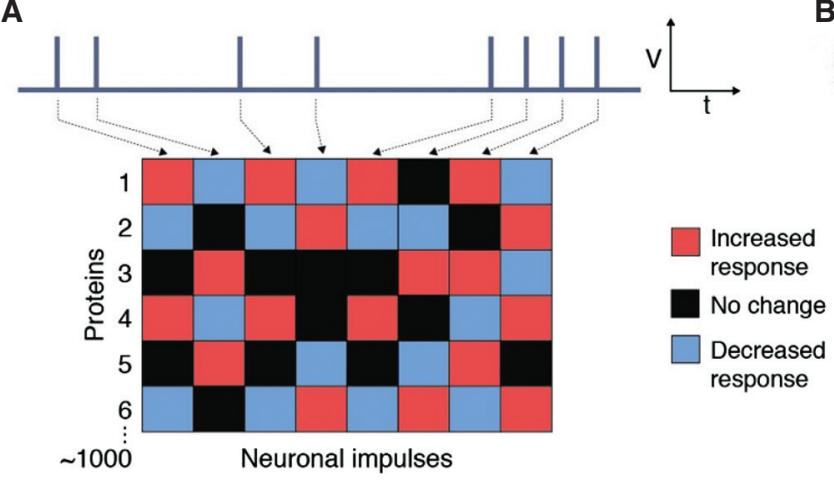

B

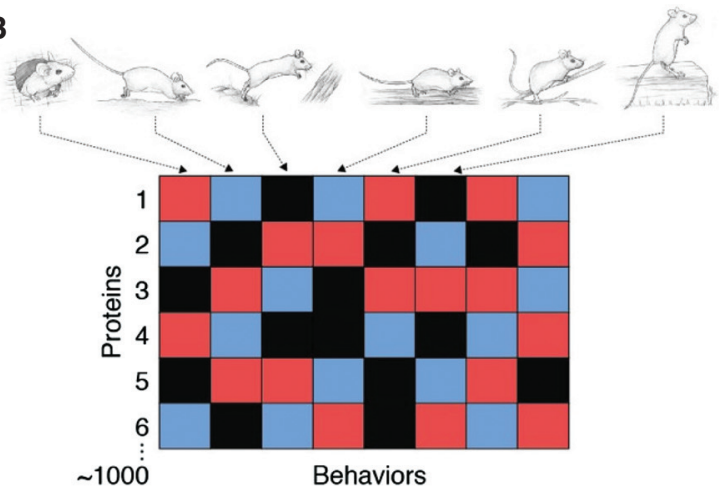

Figure 3. Postsynaptic protein combinations control responses to patterns of neural activity and behavioral responses. ( $A$ ) The vertical column shows the role that each of six postsynaptic proteins (out of 1000 or more PSP proteins) play in the response to a neural impulse in a train of eight pulses. (V) Voltage, (t) time. The color-coded key indicates the direction of the modulation of the EPSC magnitude. $(B)$ Combinations of PSP proteins modulate the magnitude of behavioral responses when animals are in different environments.

\section{The PSP Controls the Innate and Learned Behavioral Repertoire}

In mice and humans, mutations in PSP genes lead to many behavioral phenotypes in innate and learned behaviors. We performed a large-scale study of innate and learned behavior in mice carrying mutations in PSP genes and found that each behavior was controlled by a combination of PSP proteins (Komiyama et al. 2018). Each combination included proteins that were required to amplify or attenuate the respective behavior, revealing that the combinatorial mechanism tunes the magnitude of behavioral responses (Fig. 3B). The fact that innate and learned behaviors used the same combinatorial mechanism indicates that these classes of behavior are "encoded" by a common molecular and physiological mechanism. A striking feature of this combinatorial molecular mechanism is that it could be responsible for generating a vast number of different behaviors. The genes with the strongest phenotypes were those that encoded proteins found in PSD95 supercomplexes, again supporting the view that these are important molecular machines in the postsynaptic terminal.

A key feature of the connectionist theory is that long-term synaptic strength is a correlate of the behavioral magnitude and that increasing synaptic strength causes learning. However, there have been many studies showing that interference with synaptic proteins results in a dissociation between synaptic strength and learning (Bannerman et al. 1995, 2014; Hölscher 1997; Migaud et al. 1998; Zamanillo et al. 1999; Sanders and Fanselow 2003; Uekita and Okaichi 2005; Horner et al. 2018). In our large-scale genetic study of PSP proteins (Komiyama et al. 2018; Kopanitsa et al. 2018), we asked if the magnitude or direction of synaptic potentiation in the hippocampal CA1 region correlated with hippocampus-dependent forms of learning and found a robust dissociation, indicating that there is not a causal relationship. These results pose a significant challenge to the connectionist theory's explanation of learning and suggest that the PSP uses other physiological mechanisms to store and recall information that controls behavior.

\section{The PSP Has Ancient Origins Predating the Neuron}

The characterization of the PSP paved the way for the first systematic analysis of synapse evolution, and this led to questions about the central role of neurons and synaptic weight in behavior posited by the connectionist theory. We found that all major classes of synaptic protein evolved before the origin of neurons in unicellular organisms (Emes et al. 2008; Ryan and Grant 2009; Emes and Grant 2011, 2012; Bayés et al. 2017). The most ancient synaptic molecular machinery is postsynaptic proteins, which can be traced to the very earliest prokaryotic life-forms that lived $\sim 3.5$ billion years ago. These proteins include membrane-associated multiprotein signaling complexes - from which PSD95 supercomplexes evolved - and they control how prokaryotes respond to chemical signals and generate their diverse behavioral responses including spatial navigation. Remarkably, these ancient bacterial complexes achieve this by measuring the time interval between stimuli, which is the same function performed by vertebrate PSD95 supercomplexes in synapses, which are also required for spatial navigation in mice (Migaud et al. 1998). These findings are important because they point to the primacy and conservation of the PSP and multiprotein complexes over billions of years and across many phyla in which they control temporal integration of signals. Thus, the evolutionary studies support the view that the most fundamental building blocks of the behavioral repertoire are multiprotein signaling complexes and not the excitable neuron of the connectionist theory.

The evolutionary studies also revealed that there was a step increase in the complexity of the PSP early in the vertebrate lineage. This increase was generated by two whole-genome duplications 550 million years ago and expanded each protein family (Emes et al. 2008; Ryan et al. 2008, 2013; Ryan and Grant 2009; Emes and Grant 2011, 2012; Nithianantharajah et al. 2013; Bayés et al. 2017). We showed that this increase in molecular complexity led to an increase in the complexity of the mammalian behavioral repertoire, a greater complexity in synapse responses to patterns of activity, and an increase 
in synapse diversity and complexity in the synaptome maps (Nithianantharajah et al. 2013; Ryan et al. 2013; Komiyama et al. 2018; Kopanitsa et al. 2018; Zhu et al. 2018). These findings underscore the view that PSP complexity and synaptome map architecture are the substrate for the behavioral repertoire. In the vertebrate lineage leading to teleost fish (including zebrafish) there was an additional genome duplication that generated distinct synapse types and functions (Bayés et al. 2017).

\section{DEFINING FEATURES OF THE SYNAPTOMIC THEORY OF BEHAVIOR}

Integrating the insights described above into a single unifying theory presented a challenge, but a common thread in all the biochemical, neuroanatomical, electrophysiological, and behavioral data sets was evidentthat particular combinations of PSP proteins contribute to specific structures and functions. Recognizing that combinations of proteins build complexes and supercomplexes and these are then distributed into different synapse types provides a structural foundation for the physiological and behavioral functions (Fig. 1). Furthermore, because synapses composed of different proteins have different physiological responses to patterns of activity, the activity patterns associated with any given behavior will preferentially drive synapses in particular neurons, and these neurons can generate the relevant behavioral output. Hence, as a result of the molecular structural foundations, different activity patterns and behaviors will use particular combinations of proteins. It follows that the spatial distribution of synapse types within synaptome maps must be a mechanism for storing or representing innate or learned behaviors in different regions of the brain. The molecular evolutionary data also fits well within this framework, as the increase in vertebrate proteome complexity and synapse diversity would be expected to contribute to the greater behavioral complexity of vertebrates that has been observed. From these broader considerations, we can summarize the following specific features of the synaptomic theory:

- Information is "written" in the PSP of individual synapses.

- Information can be "read" or recalled from a synapse by the instantaneous modulation of responses occurring during patterns of activity.

- The complexity of the PSP endows each synapse with the ability to discriminate many patterns of activity, including complex trains.

- Synapses with different PSPs are specialized to maximally respond to (or select) specific patterns of activity.

- Synapse diversity is defined by combinations of PSP proteins and therefore information storage and recall are also defined by the same molecular combinatorial principles.

- Synapse diversity results from a series of genetic programs that assemble and distribute combinations of in- dividual proteins, complexes, and supercomplexes into different synapses.

- Synaptome maps describe the spatial organization and architecture of synapse diversity.

- A synaptome map can generate many spatiotemporal outputs from incoming patterns of activity.

- The information stored in synaptome maps can be accessed by activity in local connected circuits or in widely distributed neuronal networks.

- The retrieval of information from synaptome maps can be suppressed by LTP of synaptic transmission.

- Mutations that change synapse diversity and synaptome maps result in altered spatiotemporal outputs in response to patterns of activity, thereby changing behavioral responses including those in genetic diseases.

In the following sections, I will illustrate how the synaptomic theory explains physiological and behavioral functions and will contrast some of the key differences in the synaptomic and connectionist theories. This contrast is not meant to imply that the synaptomic theory should supplant the connectionist theory, as, indeed, the two theories can coexist.

For the purposes of simplification and illustration, the synaptome in the connectionist theory is composed of similar synapses, whereas synapses are diverse in the synaptomic theory. The synapses in the connectionist theory are referred to as "stable" and those in the synaptomic theory as "dynamic," meaning that their primary physiological function with respect to behavior is the maintenance of stable synaptic strength or dynamic instantaneous modulation (STP), respectively. Hence, the contrast will be between stable-similar and dynamic-diverse synapses.

\section{THE RESPONSE OF A SYNAPTOME MAP TO PATTERNS OF ACTIVITY}

Figure 4 illustrates the different outputs (excitatory postsynaptic current [EPSC]) of stable and dynamic synapses when presented with a train of activity. Note that the stable synapse maintains the same strength (EPSC amplitude) for the different pulses, whereas the dynamic synapses vary their strength depending on the interval of time from the preceding impulse. Furthermore, three dynamic synapse types (with different proteomes) are depicted that have different basal synaptic strength (indicated in the amplitude of the first EPSC response) and show differing strengths in response to the same temporal pattern. Thus, synapse molecular diversity gives different response profiles to the same pattern of activity. It also follows that the response profile reveals the molecular composition of the synapse proteome. In other words, the physiological response to a pattern of activity in dynamic synapses is a readout of the information stored in the proteome of the synapse.

Next, we will take these synapses and add the spatial dimension that is inherent in synaptome maps by creating a synaptome map of nine synapses (Fig. 5). The map depicted for the stable synapses contains nine synapses 


\section{Presynaptic input}
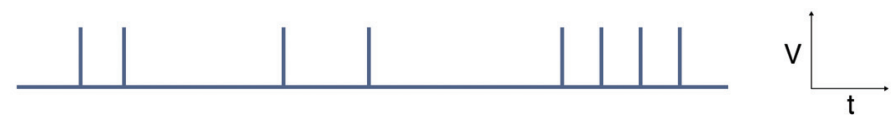

\section{Postsynaptic output}

Stable and similar synapse
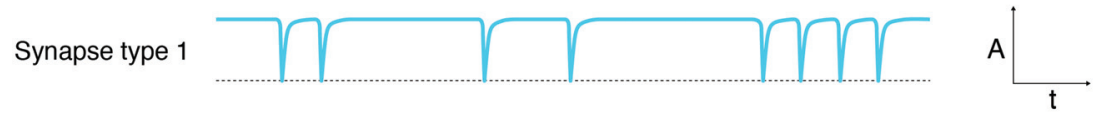

Dynamic and diverse synapses

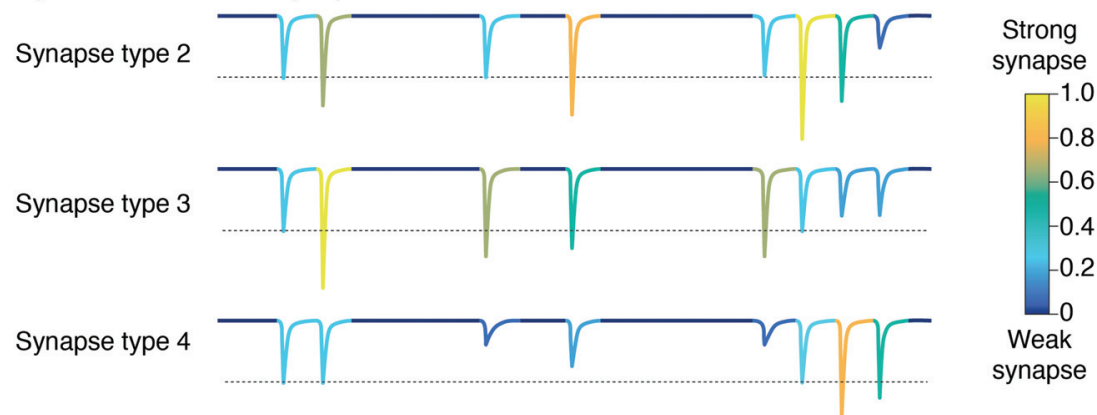

Figure 4. The response of stable and dynamic synapses to patterns of activity. The pattern of activity in the presynaptic axon is shown as a train of eight impulses (top). The postsynaptic output is the amplitude of the excitatory postsynaptic current. The amplitude of the stable synapse responses is invariant throughout the activity pattern, whereas the dynamic synapse's output is dynamic and varies. Three subtypes of dynamic synapses are shown, each with a different response profile to the pattern of activity. The color key shows the magnitude of the response and corresponds to colors in Figure 5.

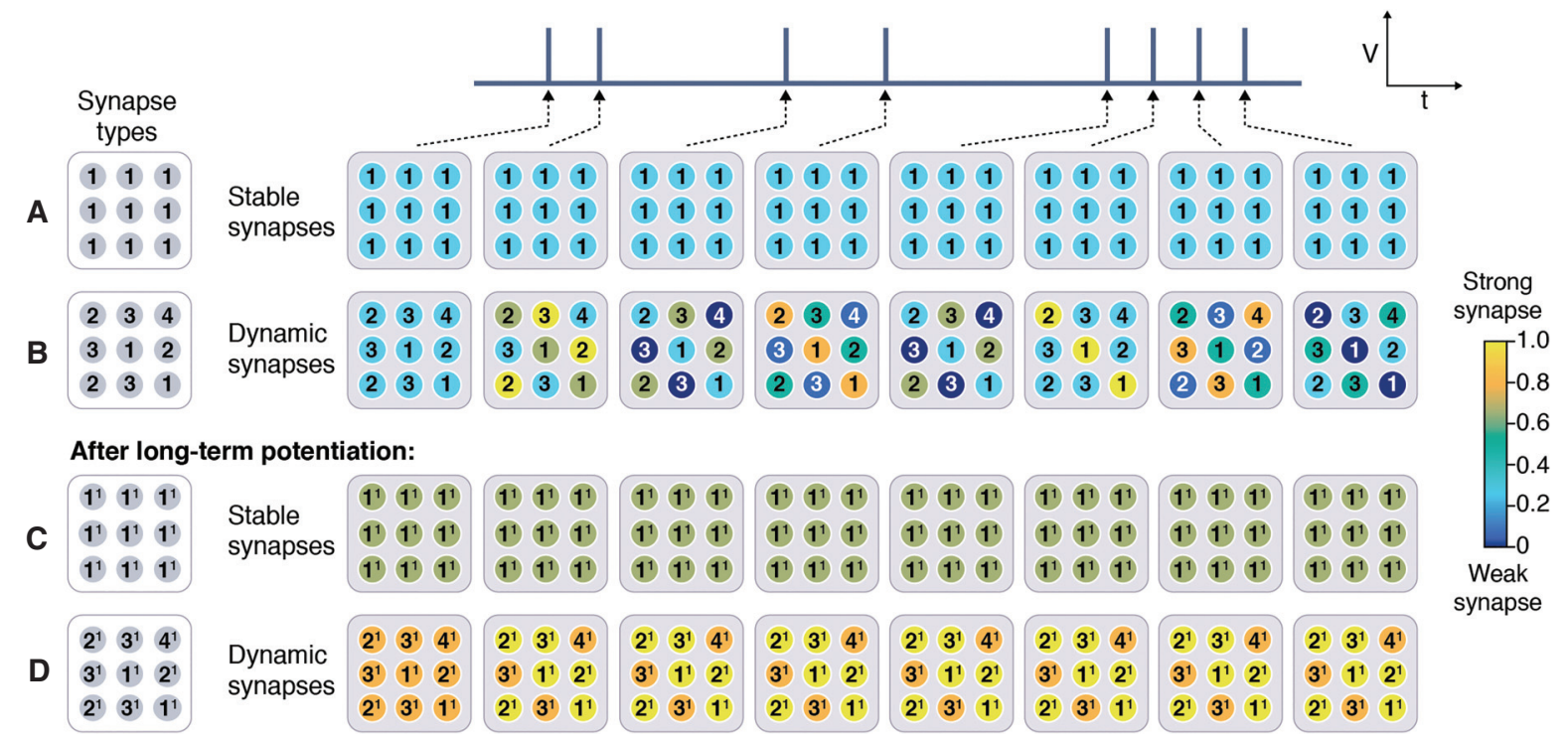

Figure 5. Spatiotemporal responses of synaptome maps to patterns of activity. Synaptome maps made from nine synapses are shown. ( $A$ ) A synaptome map composed of stable-similar synapses (Type 1) produces invariant postsynaptic responses to the pattern of activity (corresponds to that shown in Fig. 4). (B) A synaptome map made from dynamic-diverse synapses (Types 2-4) produces a changing spatiotemporal output in response to the pattern of activity. $(C)$ The stable-similar synaptome map after LTP (potentiated synapses shown with superscript 1) produces invariant postsynaptic responses, similar to the unpotentiated synaptome in $A$. $(D)$ The dynamicdiverse synaptome map after LTP shows a spatiotemporal output that has an attenuated range of amplitudes and therefore less information. Note that potentiated Type 2 and 3 synapses show differential responses before LTP $(A)$ and the same response after LTP $(D)$. 
that are all similar (type 1) (Fig. 5, row A). The response to the pattern of activity (same as in Fig. 4) shows an invariant EPSC amplitude, consistent with their stability. In contrast, a synaptome map made from three dynamicdiverse synapse types (types 2-4) (Fig. 5, row B) shows a varying spatiotemporal pattern. The differences between the outputs of synaptome maps composed of stable-similar and dynamic-diverse synapses are striking: There is a lack of spatiotemporal information from the stable-similar synaptome and a rich spatiotemporal output from the dynamic-diverse synaptome. This reveals that synaptome maps of dynamic-diverse synapses store more information than stable-similar synapses. The temporal dimension is particularly interesting because the stable synaptome outputs are invariant and can be considered as "snapshots," whereas the changing output in dynamic-diverse synaptomes generates a "movie" from the sequence of changing snapshots.

The spatiotemporal dynamic output of dynamic-diverse synaptomes has at least three important functional implications. First, a synaptome of dynamic-diverse synapses can store many more snapshots or representations than can a synaptome of stable-similar synapses. Second, these many representations can be multiplexed into a single synaptome map - the dynamic-diverse synaptome serves as a library of snapshots or representations. Third, the representations within this library can be recalled in a sequence that is determined by the pattern of activity. Each of these snapshots could drive a different motor action and hence the dynamic-diverse synaptome map could effectively program a sequence of behaviors (Fig. 6).

Another important feature of this theory is that it provides a very simple recall or memory retrieval mechanism. This is very appealing because there is a simple and direct relationship between information storage and recall: The information is stored in the synapse proteomes, and it is recalled by the pattern of neural activity. Or more broadly, information is written in the molecular composition of diverse synapses, and this information is read by the dynamic properties of the synapses in response to patterns of activity. Thus, the synaptomic theory provides a straightforward explanation for the recall of innate or learned behaviors and the generation of behavioral sequences, which are two fundamental properties of behavior that are not readily explained in the connectionist theory.

\section{LEARNING AND LTP IN THE SYNAPTOMIC THEORY}

There is a literature showing that learning induces changes in either the abundance or posttranslational modification of synapse proteins (for a review, see Asok et al. 2019). Any change in a synaptic protein that alters the dynamic response of a synapse will alter the spatial and temporal output of the synaptome. Unlike the connectionist theory, there is no need to change stable synaptic strength to store a memory in the synaptomic theory. Furthermore, as described in the previous section, a change in the proteome of synapses that occurs with learning could program a novel behavioral sequence, such as a set of new motor actions. In contrast with this simple and potentially very versatile mechanism, it is unclear how the connectionist theory can explain how a sequence of motor actions can be learned without invoking the formation of new sets of circuits for each step in the motor sequence, which would likely require major structural reorganization of brain. The connectionist theory relies on static synaptic weight and does not have a temporal dimension.

A central tenet of the connectionist theory is that learning generates a change in the stable synaptic strength between neurons, either by an LTP-like process or by an increase in the number of connections (Asok et al.

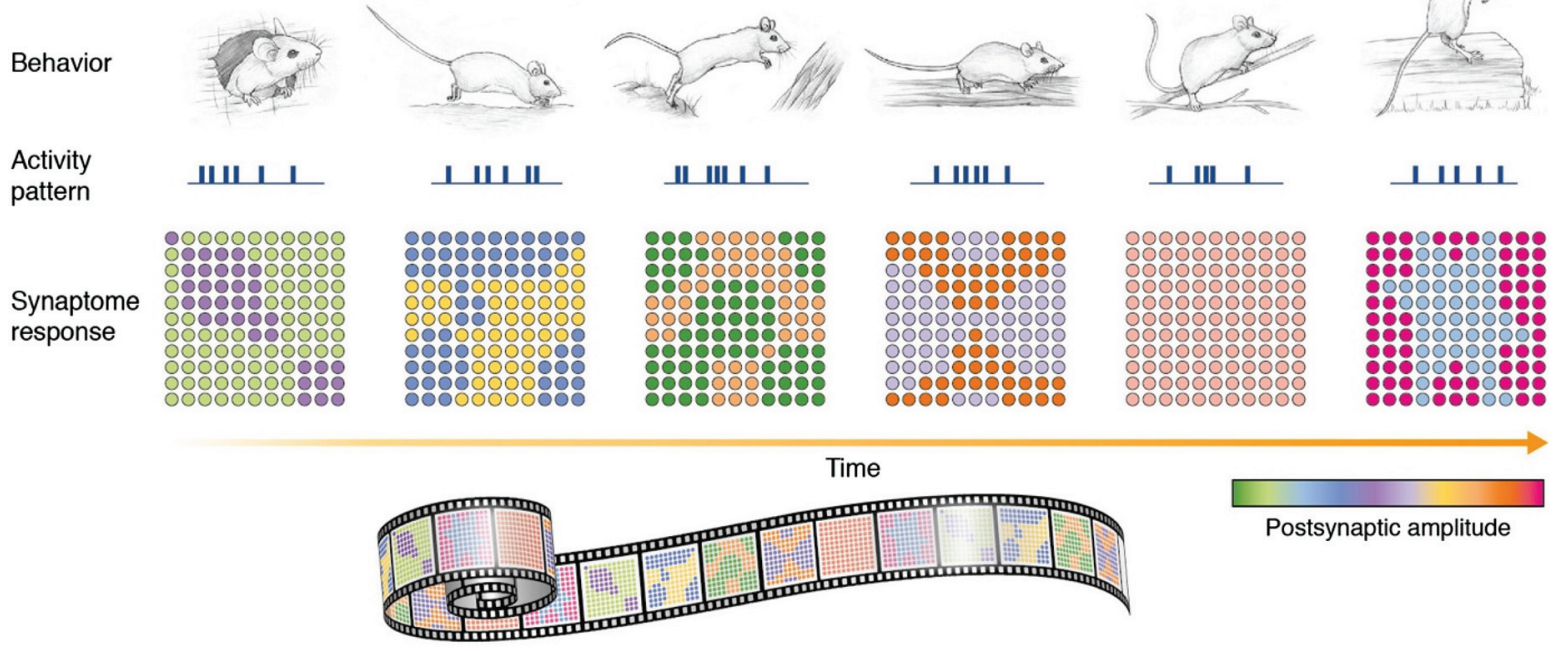

Figure 6. Synaptome maps continuously change as different sensory inputs generate different patterns of activity, akin to a movie of representation in the brain. (Adapted from Zhu et al. 2018.) 
2019). In the synaptomic theory, there is no need for these mechanisms as a change in the synapse proteome that changes the dynamic properties of the synapse is sufficient. This leads to the question: What might LTP do in the synaptomic theory? LTP is defined by an increase in the baseline stable synaptic strength, and this increase is associated with a diminished variance in the EPSC amplitude (Bekkers and Stevens 1990; Malinow and Tsien 1990; Kullmann and Nicoll 1992), We can then depict the effects that LTP will have on the output of synaptome maps exposed to patterns of activity in Figure 5C,D.

First, we will consider the effects of LTP on the stable-similar synaptome (Fig. 5A), where the potentiated synapses are indicted by the superscript 1 (Fig. 5C). The potentiated synaptome's spatiotemporal response to the pattern of activity is identical to that of the unpotentiated synaptome, only the amplitude is greater. Next, consider the effect of potentiation on synaptomes composed of dynamic-diverse synapses (Fig. 5B,D): In addition to the increase in amplitude of the responses, there is a reduction in variance with the result that the differences between the diverse synapses is diminished, effectively reducing the spatiotemporal output of the synaptome map. LTP is effectively masking the information within the dynamic-diverse synaptome map and thus reducing the capacity to retrieve the information stored in the molecular composition of the synapse proteome. Thus, in the synaptomic theory, LTP is a modulatory mechanism that gates the retrieval or recall of behavioral representations stored in synaptomes. This view that LTP inhibits learning and recall is consistent with experimental evidence (McNaughton et al. 1986; Moser et al. 1998).

\section{BUILDING A REPERTOIRE OF BEHAVIORS}

All animals use a diverse repertoire of behavioral responses to varied and changing environments. It has long been accepted that there are individual behavioral components and indeed nineteenth century naturalists and psychologists recognized that the behavioral repertoire of animals had an organization or structure in which elementary behaviors, such as reflexes, were basic building blocks for more complex instincts, higher forms of cognition, and ultimately consciousness (Spencer 1870; Darwin 1872; Romanes 1882; James 1890; Morgan 1891). The basic building blocks of the behavioral repertoire were further classified into innate (inherited) or learned responses (Tinbergen 1951; Lorenz 1965). There is also the necessity of deploying these behavioral components in an appropriate temporal sequence so as to produce relevant, coordinated action patterns. As illustrated in Figure 6, a mouse receives novel sensory inputs as it moves through its environment, and these generate distinct patterns of neural activity that trigger a continuously changing spatiotemporal output from a synaptome map, which could generate the behavioral sequence.

In addition to the capacity of a synaptome map to represent many different behaviors and behavioral sequences, the diversity of synaptome maps in specialized brain re-
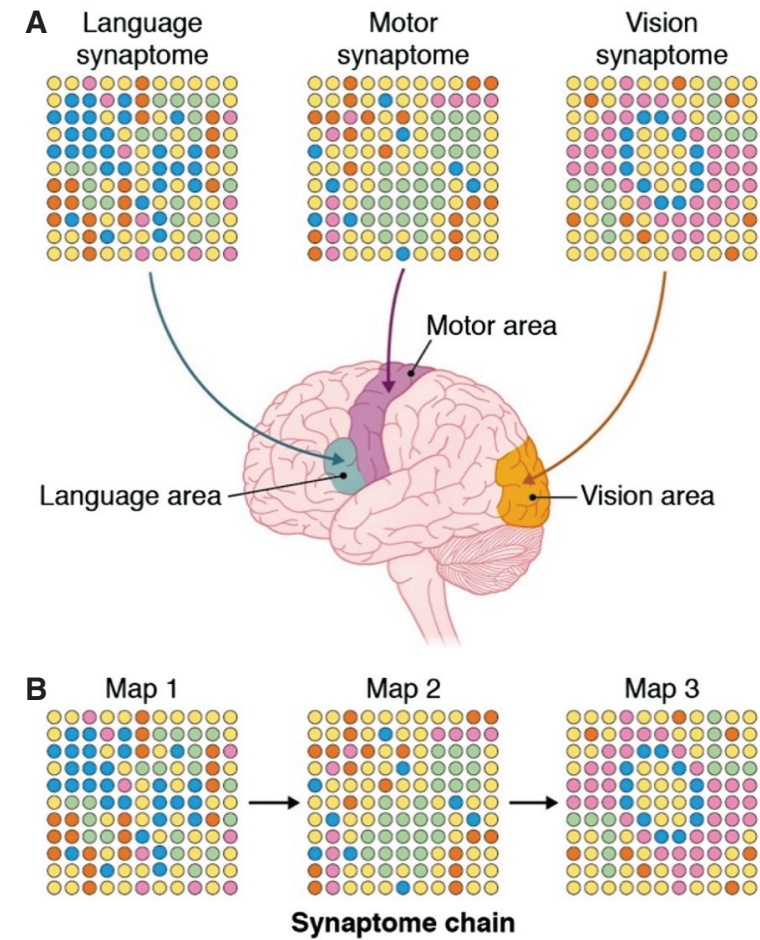

Figure 7. Synaptome maps are specialized in different brain regions. $(A)$ The specialization of synaptome maps allow those regions to generate distinct responses to patterns of activity. $(B) \mathrm{A}$ hypothetical chain of connected synaptomes in which the neurons of map 1 drive map 2 and these in turn drive map 3. A chain of maps could program complex sequences of behavior.

gions offers further opportunities to build a behavioral repertoire (Fig. 7A). We found that each brain region has a particular synaptome map (Zhu et al. 2018), and these distinct maps could contribute to different behaviors. In other words, synaptome maps in different neurons or brain regions that are made from different synapses could "build" an animal's behavioral repertoire. The specialization of the synaptome in brain regions could arise during evolution by minor changes in gene regulation (e.g., point mutations in enhancer regions of genes) that change the expression of synaptic proteins, which would confer new behaviors. Thus, molecular evolution, rather than growth of new circuits as posited in the connectionist theory, could create or modify the behavioral repertoire.

\section{MUTATION AND DISEASE IN THE SYNAPTOMIC THEORY}

The synaptome architecture is based on the hierarchical structural organization of combinations of proteins into complexes and supercomplexes and their distribution into synapses within a synaptome map (Frank et al. 2016, 2017; Frank and Grant 2017; Zhu et al. 2018), and this is the substrate for the physiological responses to patterns of neuronal activity. Therefore, mutations that interfere with the mechanisms controlling protein organization and distribution will result in different synaptome maps. 
We established that mouse genetic models of schizophrenia and autism/intellectual disability result in widespread changes in the synaptome in many brain regions (Zhu et al. 2018). In addition to the direct impact of the loss of a particular protein on synapse diversity and synaptome maps, we discovered "synaptome reprogramming," which is the change in synapse proteins in response to mutations in other genes. Our results suggest that altered synaptome maps will be a general feature of most — if not all — genetic brain disorders.

Synaptome mapping of different mutations will be important for comparing and contrasting different diseases. It might be expected that convergent phenotypes in polygenic disorders such as autism and schizophrenia will be detected using synaptome mapping. These convergent phenotypes might be observed in selected types of synapses, brain regions, or cell types, all of which can be detected using synaptomic technology. There are approximately 1500 postsynaptic proteins in human synapses, and hundreds of their cognate genes are mutated in diseases (Bayés et al. 2011). In principle, it will be possible to categorize these diseases according to the similarity in their synaptomes.

Synapse diversity raises the concept that each disease will have susceptible and resilient synapse subtypes. With the development of tools for synaptome mapping using multiple synapse protein markers, it will be feasible to quantify the susceptible and resilient synapse subtypes in diseases. Indeed, we expect that different diseases will be characterized by "signatures" of subtype pathology.

Synaptome maps offer a new perspective on Alzheimer's disease and other neurodegenerative disorders, too, as these are diseases characterized by focal damage (e.g., plaques). The accumulation of these focal lesions will cause local loss of synapses and gradually degrade the synaptome map. The functional consequences of this will be to reduce the capacity of the map to represent behaviors with high fidelity, which may be relevant to the failure of memory recall or confusion that occurs in these disorders.

A future direction of research, with potential clinical impact, will be to develop brain imaging tools for measuring the synaptome in living individuals. The feasibility of this approach is supported by correlations between functional magnetic resonance imaging (fMRI) and the synaptome and synapse proteomes in brain regions of mice and humans (Roy et al. 2018b; Zhu et al. 2018).

\section{EVOLUTION IN THE SYNAPTOMIC THEORY}

A key feature of the synaptomic theory is the complexity of the proteome of individual synapses, as this confers the molecular computational properties that underlie the capacity to detect and discriminate patterns of neural activity. The expansion in the synapse proteomes was driven by genome duplications that generated paralog diversity and expanded the number of complexes and supercomplexes, thereby creating an opportunity for vertebrates to expand their synapse diversity. We mapped the synaptomes of two postsynaptic paralogs, PSD95 and SAP102, and found distinct and overlapping expression at the individual synapse level, thereby confirming that these molecular evolutionary events increased synapse diversity and the complexity of synaptome maps (Zhu et al. 2018). Our characterization of behavioral and physiological phenotypes in mice carrying mutations in paralogs strongly supports the conclusion that genomic evolution drove synapse proteome and synaptome complexity, with the result of generating the more complex higher cognitive functions that characterize vertebrate species (Nithianantharajah et al. 2013; Ryan et al. 2013).

Synaptome mapping of different species - comparative synaptomics - will likely be important for understanding animal behavior and the utility of species as models of human disease. Humans and mice diverged from a common ancestor $\sim 90$ million years ago and share a conserved brain architecture, which can now be mapped at single-synapse resolution using synaptomic methods. Such species comparisons using synaptome mapping could provide unique insights, including whether there are species-specific synapse types and if the specialization of brain regions, such as human language regions, show particular synapse compositions.

Our understanding of synapse molecular evolution is also relevant to the prediction made by the synaptomic theory that LTP is a modulatory mechanism that gates the retrieval of information stored in synaptomes because the dynamic properties of synapses arose from the ancient signaling complexes present in prokaryotes (Emes et al. 2008; Emes and Grant 2011, 2012), several billions of years before LTP in metazoan synapses. LTP is therefore a late-evolved mechanism that modulates a more ancient and fundamental mechanism of behavior.

\section{SYNAPTOME MAPPING AND NEW BEHAVIORAL VISTAS}

In principle, synaptomic theory could account for many features of behavior, including some that are presently mysterious. Specializations of synaptome maps in different regions of the brain (Roy et al. 2018a,b; Zhu et al. 2018) would be expected to provide the specialized functions ascribed to those regions (Fig. 7). For example, the language regions of the human neocortex have a specific PSP composition (Roy et al. 2018b). Language could be encoded in the synaptome maps in this region and the input of patterns of nerve impulses originating from the auditory system could induce sequences of synaptome outputs that correspond to the perceptual building blocks of language - namely, phonemes and words. Consider the illustration in Figure 6, but replace each mouse behavior with a particular sound, and each synaptome response would represent a phoneme, and the sequence of these in the "movie" could generate the representation of words and phrases. In this way, the synaptome map could encode the representation of language. This is an appealing model as (i) the genetic mechanisms that define a "language synaptome map" could account for the observation that 
there is an innate and universal language capacity (in humans) (Chomsky 1967); (ii) a developmental increase in synapse diversity could explain the increase in vocabulary and syntactical complexity that occurs during childhood; and (iii) experience-dependent changes in the language synaptome map could, in principle, explain the acquisition of specific languages.

It is also interesting to consider that multiple synaptome maps could be connected into a chain and the output of this chain could generate more complex and diverse temporal responses, which might underpin longer and more complex behavioral sequences (Fig. 7B). It is implicit in the synaptomic theory that the hierarchical molecular properties that build a synaptome map would themselves be a higher-order building block for anatomical hierarchies based on the connections between these maps. This molecule-to-system hierarchical model has small-world network topology at multiple levels, including the molecular networks (Pocklington et al. 2006; Roy et al. 2018a,b) and the anatomical synaptome architecture at the systems neuroscience level (Zhu et al. 2018). It will be of great interest to dissect the genetic mechanisms that influence these different levels of the hierarchical architecture as they may underpin the logical framework, the constraints, and the species differences in the behavioral repertoire. It follows that the molecular regulatory mechanisms that control the levels of the hierarchy will be disrupted in neurological diseases, which will manifest with a pattern of behavior defined by the reprogrammed synaptome architecture.

\section{CONCLUSION}

This article outlines a new theory for behavior based on the complexity of the PSP, synapse diversity, and the dynamic physiological properties of synapses in response to patterned activity. The framework and logic for the synaptomic theory is based on a "bottom-up" understanding of the molecular basis of synapse diversity, its spatial organization into synaptome maps, and the molecular mechanisms responsible for detecting and discriminating patterns of activity. Synaptome maps comprising dynamic-diverse synapses have the capacity to store information including temporal sequences, which may underlie behavioral and perceptual sequences. Although there are stark contrasts in the fundamental principles of the synaptomic and connectionist theories, they are by no means mutually exclusive, and it will be important to integrate these two models. For example, we need to understand more about the synapse diversity on individual neuron types, how defined connected circuits in which neurons and dendrites having diverse synapses can function, and how neuromodulatory processes and other forms of synaptic plasticity gate the function of synaptomes built from dynamicdiverse synapses.

A key feature of the synaptomic theory is that it is grounded in the genome and proteome, which provides a direct path from the hundreds of genetic diseases that affect synapses to the mechanisms of behavioral change observed in these disorders. The connectome and connectionist theory currently lacks this molecular logic and the power to explain genetic disease phenotypes. A potentially more profound contribution of synaptomic theory is that it outlines a clear path from genes to the physiological mechanisms of information processing in an evolutionary framework. By integrating the mechanisms of genome evolution, synaptomic theory provides a mechanism for the evolution of behavior that can be rigorously quantified and studied using molecular methods. The molecular synaptic mechanisms within this theory are not restricted to organisms with nervous systems and thus the theory spans all organisms to the last universal common ancestor $\sim 3.5$ billion years ago.

This outline of synaptomic theory suggests many new kinds of experiments and tests of the theory. Current synaptomic methods have opened a new window on the complexity of the brain and suggest exciting and unexplored possibilities conferred by synapse diversity and synaptome architecture. There is a pressing need to develop new methods for the simultaneous study of the molecular and physiological properties of single synapses in tissue and the intact behaving animal. The vast number of synapses is a hallmark of the brain, and the capacity to study them in large numbers using systematic methods is a new and important "omic" frontier in neuroscience.

\section{ACKNOWLEDGMENTS}

I am grateful for the contributions of and conversations with all of my laboratory members and collaborators who have contributed to the described experiments that have underpinned this theory and to Erik Fransén, Noboru Komiyama, and Tom O'Dell for comments on the manuscript. This work was supported by the Medical Research Council (M501682, G0802238), the Wellcome Trust (202932), the European Union Seventh Framework Programme (HEALTH-F2-2009-241498 EUROSPIN, HEALTH-F2-2009-242167 SynSys, HEALTH-F2-2009241995 GENCODYS) and the European Research Council (ERC) under the European Union's Horizon 2020 research and innovation programme (695568 SYNNOVATE).

\section{REFERENCES}

Abbott LF, Regehr WG. 2004. Synaptic computation. Nature 431: 796-803. doi:10.1038/nature03010

Adrian EDA. 1928. The basis of sensation, the action of the sense organs. W.W. Norton, New York.

Asok A, Leroy F, Rayman JB, Kandel ER. 2019. Molecular mechanisms of the memory trace. Trends Neurosci 42: 1422. doi:10.1016/j.tins.2018.10.005

Bannerman DM, Good MA, Butcher SP, Ramsay M, Morris RG. 1995. Distinct components of spatial learning revealed by prior training and NMDA receptor blockade. Nature 378: 182 186. doi:10.1038/378182a0

Bannerman DM, Sprengel R, Sanderson DJ, McHugh SB, Rawlins JN, Monyer H, Seeburg PH. 2014. Hippocampal synaptic plasticity, spatial memory and anxiety. Nat Rev Neurosci 15: 181-192. doi:10.1038/nrn3677

Bayés À, van de Lagemaat LN, Collins MO, Croning MD, Whittle IR, Choudhary JS, Grant SG. 2011. Characterization of the 
proteome, diseases and evolution of the human postsynaptic density. Nat Neurosci 14: 19-21. doi:10.1038/nn.2719

Bayés À, Collins MO, Croning MD, van de Lagemaat LN, Choudhary JS, Grant SG. 2012. Comparative study of human and mouse postsynaptic proteomes finds high compositional conservation and abundance differences for key synaptic proteins. PLoS One 7: e46683. doi:10.1371/journal.pone .0046683

Bayés À, Collins MO, Reig-Viader R, Gou G, Goulding D, Izquierdo A, Choudhary JS, Emes RD, Grant SG. 2017. Evolution of complexity in the zebrafish synapse proteome. Nat Commun 8: 14613 . doi: $10.1038 /$ ncomms 14613

Bekkers JM, Stevens CF. 1990. Presynaptic mechanism for longterm potentiation in the hippocampus. Nature 346: 724-729. doi:10.1038/346724a0

Chomsky N. 1967. Recent contributions to the theory of innate ideas: summary of oral presentation. Synthese 17: 2-11. doi:10 .1007/BF00485013

Coba MP, Pocklington AJ, Collins MO, Kopanitsa MV, Uren RT, Swamy S, Croning MD, Choudhary JS, Grant SG. 2009. Neurotransmitters drive combinatorial multistate postsynaptic density networks. Sci Signal 2: ra19. doi:10.1126/scisignal .2000102

Collins MO, Yu L, Coba MP, Husi H, Campuzano I, Blackstock WP, Choudhary JS, Grant SG. 2005. Proteomic analysis of in vivo phosphorylated synaptic proteins. $J$ Biol Chem 280: 5972-5982. doi:10.1074/jbc.M411220200

Collins MO, Husi H, Yu L, Brandon JM, Anderson CN, Blackstock WP, Choudhary JS, Grant SG. 2006. Molecular characterization and comparison of the components and multiprotein complexes in the postsynaptic proteome. J Neurochem 97 (Suppl 1): 16-23. doi:10.1111/j.1471-4159.2005.03507.x

Darwin C. 1872. The expression of the emotions in man and animals. John Murray, London.

Distler U, Schmeisser MJ, Pelosi A, Reim D, Kuharev J, Weiczner R, Baumgart J, Boeckers TM, Nitsch R, Vogt J, et al. 2014. In-depth protein profiling of the postsynaptic density from mouse hippocampus using data-independent acquisition proteomics. Proteomics 14: 2607-2613. doi:10.1002/pmic .201300520

Dittman JS, Kreitzer AC, Regehr WG. 2000. Interplay between facilitation, depression, and residual calcium at three presynaptic terminals. J Neurosci 20: 1374-1385. doi:10.1523/ JNEUROSCI.20-04-01374.2000

Emes RD, Grant SG. 2011. The human postsynaptic density shares conserved elements with proteomes of unicellular eukaryotes and prokaryotes. Front Neurosci 5: 44. doi:10.3389/ fnins.2011.00044

Emes RD, Grant SG. 2012. Evolution of synapse complexity and diversity. Annu Rev Neurosci 35: 111-131. doi:10.1146/ annurev-neuro-062111-150433

Emes RD, Pocklington AJ, Anderson CN, Bayes A, Collins MO, Vickers CA, Croning MD, Malik BR, Choudhary JS, Armstrong JD, et al. 2008. Evolutionary expansion and anatomical specialization of synapse proteome complexity. Nat Neurosci 11: 799-806. doi:10.1038/nn.2135

Fernández E, Collins MO, Uren RT, Kopanitsa MV, Komiyama $\mathrm{NH}$, Croning MD, Zografos L, Armstrong JD, Choudhary JS, Grant SG. 2009. Targeted tandem affinity purification of PSD95 recovers core postsynaptic complexes and schizophrenia susceptibility proteins. Mol Syst Biol 5: 269. doi:10.1038/ msb.2009.27

Fernández E, Collins MO, Frank RAW, Zhu F, Kopanitsa MV, Nithianantharajah J, Lemprière SA, Fricker D, Elsegood KA, McLaughlin CL, et al. 2017. Arc requires PSD95 for assembly into postsynaptic complexes involved with neural dysfunction and intelligence. Cell Rep 21: 679-691. doi:10.1016/j.celrep .2017.09.045

Frank RA, Grant SG. 2017. Supramolecular organization of NMDA receptors and the postsynaptic density. Curr Opin Neurobiol 45: 139-147. doi:10.1016/j.conb.2017.05.019

Frank RA, Komiyama NH, Ryan TJ, Zhu F, O’Dell TJ, Grant SG. 2016. NMDA receptors are selectively partitioned into com- plexes and supercomplexes during synapse maturation. Nat Commun 7: 11264. doi:10.1038/ncomms 11264

Frank RAW, Zhu F, Komiyama NH, Grant SGN. 2017. Hierarchical organization and genetically separable subfamilies of PSD95 postsynaptic supercomplexes. $J$ Neurochem 142: 504-511. doi:10.1111/jnc. 14056

Hebb DO. 1949. The organization of behavior; a neuropsychological theory. Wiley, New York.

Hölscher C. 1997. Long-term potentiation: a good model for learning and memory? Prog Neuropsychopharmacol Biol Psychiatry 21: 47-68. doi:10.1016/S0278-5846(96)00159-5

Horner AE, McLaughlin CL, Afinowi NO, Bussey TJ, Saksida LM, Komiyama NH, Grant SGN, Kopanitsa MV. 2018. Enhanced cognition and dysregulated hippocampal synaptic physiology in mice with a heterozygous deletion of PSD-95. Eur J Neurosci 47: 164-176. doi:10.1111/ejn.13792

Husi H, Grant SG. 2001. Isolation of 2000-kDa complexes of $\mathrm{N}$ methyl-D-aspartate receptor and postsynaptic density 95 from mouse brain. J Neurochem 77: 281-291. doi:10.1046/j.14714159.2001.t01-1-00248.x

Husi H, Ward MA, Choudhary JS, Blackstock WP, Grant SG. 2000. Proteomic analysis of NMDA receptor-adhesion protein signaling complexes. Nat Neurosci 3: 661-669. doi:10.1038/ 76615

James W. 1890. The principles of psychology. H. Holt, New York.

Kandel ER. 1976. Cellular basis of behavior: an introduction to behavioral neurobiology. W.H. Freeman, San Francisco.

Komiyama NH, Watabe AM, Carlisle HJ, Porter K, Charlesworth P, Monti J, Strathdee DJ, O'Carroll CM, Martin SJ, Morris RG, et al. 2002. SynGAP regulates ERK/MAPK signaling, synaptic plasticity, and learning in the complex with postsynaptic density 95 and NMDA receptor. $J$ Neurosci 22: 9721-9732. doi:10.1523/JNEUROSCI.22-22-09721.2002

Komiyama NH, van de Lagemaat LN, Stanford LE, Pettit C, Strathdee DJ, Strathdee JE, Fricker DG, Tuck EJ, Elsegood KA, Ryan TJ, et al. 2018. Synaptic combinatorial molecular mechanisms generate repertoires of innate and learned behavior. bioRxiv doi:10.1101/500389

Kopanitsa MV, van de Lagemaat LN, Afinowi NO, Strathdee DJ, Strathdee KE, Fricker DG, Tuck EJ, Elsegood KA, Croning MD, Komiyama NH, et al. 2018. A combinatorial postsynaptic molecular mechanism converts patterns of nerve impulses into the behavioral repertoire. bioRxiv doi:10.1101/500447

Kullmann DM, Nicoll RA. 1992. Long-term potentiation is associated with increases in quantal content and quantal amplitude. Nature 357: 240-244. doi:10.1038/357240a0

Lorenz K. 1965. Evolution and modification of behavior. University of Chicago Press, Chicago.

Malinow R, Tsien RW. 1990. Presynaptic enhancement shown by whole-cell recordings of long-term potentiation in hippocampal slices. Nature 346: 177-180. doi:10.1038/346177a0

Markram H, Gupta A, Uziel A, Wang Y, Tsodyks M. 1998. Information processing with frequency-dependent synaptic connections. Neurobiol Learn Mem 70: 101-112. doi:10 $.1006 /$ nlme.1998.3841

McNaughton BL, Barnes CA, Rao G, Baldwin J, Rasmussen M. 1986. Long-term enhancement of hippocampal synaptic transmission and the acquisition of spatial information. $J$ Neurosci 6: 563-571. doi:10.1523/JNEUROSCI.06-0200563.1986

Migaud M, Charlesworth P, Dempster M, Webster LC, Watabe AM, Makhinson M, He Y, Ramsay MF, Morris RG, Morrison JH, et al. 1998. Enhanced long-term potentiation and impaired learning in mice with mutant postsynaptic density-95 protein. Nature 396: 433-439. doi:10.1038/24790

Morgan CL. 1891. Animal life and intelligence. Edward Arnold, London.

Moser EI, Krobert KA, Moser MB, Morris RG. 1998. Impaired spatial learning after saturation of long-term potentiation. Science 281: 2038-2042. doi:10.1126/science.281.5385.2038

Nithianantharajah J, Komiyama NH, McKechanie A, Johnstone M, Blackwood DH, St Clair D, Emes RD, van de Lagemaat LN, Saksida LM, Bussey TJ, et al. 2013. Synaptic scaffold 
evolution generated components of vertebrate cognitive complexity. Nat Neurosci 16: 16-24. doi:10.1038/nn.3276

Peng J, Kim MJ, Cheng D, Duong DM, Gygi SP, Sheng M. 2004. Semiquantitative proteomic analysis of rat forebrain postsynaptic density fractions by mass spectrometry. J Biol Chem 279: 21003-21011. doi:10.1074/jbc.M400103200

Pocklington AJ, Cumiskey M, Armstrong JD, Grant SG. 2006. The proteomes of neurotransmitter receptor complexes form modular networks with distributed functionality underlying plasticity and behaviour. Mol Syst Biol 2: 2006.0023. doi:10 1038/msb4100041

Romanes GJ. 1882. Animal intelligence. Kegan Paul, Trench, London.

Roy M, Sorokina O, McLean C, Tapia-González S, DeFelipe J, Armstrong JD, Grant SGN. 2018a. Regional diversity in the postsynaptic proteome of the mouse brain. Proteomes 6: E31. doi:10.3390/proteomes6030031

Roy M, Sorokina O, Skene N, Simonnet C, Mazzo F, Zwart R, Sher E, Smith C, Armstrong JD, Grant SGN. 2018b. Proteomic analysis of postsynaptic proteins in regions of the human neocortex. Nat Neurosci 21: 130-138. doi:10.1038/s41593017-0025-9

Ryan TJ, Grant SG. 2009. The origin and evolution of synapses. Nat Rev Neurosci 10: 701-712. doi:10.1038/nrn2717

Ryan TJ, Emes RD, Grant SG, Komiyama NH. 2008. Evolution of NMDA receptor cytoplasmic interaction domains: implications for organisation of synaptic signalling complexes. $B M C$ Neurosci 9: 6. doi:10.1186/1471-2202-9-6

Ryan TJ, Kopanitsa MV, Indersmitten T, Nithianantharajah J, Afinowi NO, Pettit C, Stanford LE, Sprengel R, Saksida LM, Bussey TJ, et al. 2013. Evolution of GluN2A/B cy- toplasmic domains diversified vertebrate synaptic plasticity and behavior. Nat Neurosci 16: 25-32. doi:10.1038/nn .3277

Sanders MJ, Fanselow MS. 2003. Pre-training prevents context fear conditioning deficits produced by hippocampal NMDA receptor blockade. Neurobiol Learn Mem 80: 123-129. doi:10 .1016/S1074-7427(03)00040-6

Segundo JP, Moore GP, Stensaas LJ, Bullock TH. 1963. Sensitivity of neurones in Aplysia to temporal pattern of arriving impulses. J Exp Biol 40: 643-667.

Skene NG, Roy M, Grant SG. 2017. A genomic lifespan program that reorganises the young adult brain is targeted in schizophrenia. eLife 6: e17915. doi:10.7554/eLife.17915

Spencer H. 1870. The principles of psychology. Williams and Norgate, London.

Tanzi E. 1893. I fatti e le induzioni dell'odierna istologia del sistema nervoso. Riv Sper Fren Med Leg 19: 419-472.

Tinbergen N. 1951. The study of instinct. Clarendon, Oxford.

Uekita T, Okaichi H. 2005. NMDA antagonist MK-801 does not interfere with the use of spatial representation in a familiar environment. Behav Neurosci 119: 548-556. doi:10.1037/ 0735-7044.119.2.548

Zamanillo D, Sprengel R, Hvalby O, Jensen V, Burnashev N, Rozov A, Kaiser KM, Köster HJ, Borchardt T, Worley P, et al. 1999. Importance of AMPA receptors for hippocampal synaptic plasticity but not for spatial learning. Science 284: 18051811. doi:10.1126/science.284.5421.1805

Zhu F, Cizeron M, Qiu Z, Benavides-Piccione R, Kopanitsa MV, Skene NG, Koniaris B, DeFelipe J, Fransén E, Komiyama NH, et al. 2018. Architecture of the mouse brain synaptome. Neuron 99: 781-799 e710. doi:10.1016/j.neuron.2018.07.007 


\section{$\$_{\text {CSH }}^{\infty}$ Cold Spring Harbor Symposia SYMPOSIA On Quantitative Biology}

\section{The Synaptomic Theory of Behavior and Brain Disease}

Seth G.N. Grant

Cold Spring Harb Symp Quant Biol 2018 83: 45-56 originally published online March 18, 2019 Access the most recent version at doi:10.1101/sqb.2018.83.037887

References This article cites 50 articles, 13 of which can be accessed free at: http://symposium.cshlp.org/content/83/45.full.html\#ref-list-1
Creative This article is distributed under the terms of the
Commons http://creativecommons.org/licenses/by/4.0/, which permits unrestricted
License reuse and redistribution provided that the original author and source are credited.

Email Alerting Receive free email alerts when new articles cite this article - sign up in Service the box at the top right corner of the article or click here. 